\title{
Introduction
}

\section{Éloquence, rhétorique, littérature. Tensions et interrogations}

Eloquence, Rhetoric, Literature. Tensions and Questions

Marion Mas, Catherine Nicolas et Anne Vibert

\section{OpenEdition}

Journals

Édition électronique

URL : https://journals.openedition.org/recherchestravaux/3854

DOI : 10.4000/recherchestravaux.3854

ISSN : 1969-6434

Éditeur

UGA Éditions/Université Grenoble Alpes

Édition imprimée

ISBN : 978-2-37747-326-7

ISSN : 0151-1874

\section{Référence électronique}

Marion Mas, Catherine Nicolas et Anne Vibert, «Introduction

Éloquence, rhétorique, littérature. Tensions et interrogations », Recherches \& Travaux [En ligne], 99 |

2021, mis en ligne le 08 décembre 2021, consulté le 11 décembre 2021. URL : http://

journals.openedition.org/recherchestravaux/3854; DOI : https://doi.org/10.4000/recherchestravaux. 3854

Ce document a été généré automatiquement le 11 décembre 2021.

(c) Recherches \& Travaux 


\title{
Introduction
}

\section{Éloquence, rhétorique, littérature. Tensions et interrogations}

\author{
Eloquence, Rhetoric, Literature. Tensions and Questions
}

\author{
Marion Mas, Catherine Nicolas et Anne Vibert
}

1 La « réactualisation de la rhétorique est directement liée aux rapports qu'une société entretient avec les questions politiques et épistémologiques ${ }^{1} »$, rappelle un article récent d'Emmanuelle Danblon. Les grandes synthèses sur l'éloquence ${ }^{2}$ ne disent pas autre chose. Dès lors, de quoi, en notre Xxi siècle commençant, le retour de l'éloquence dans l'enseignement est-il le signe? Quelles sortes de liens l'éloquence scolaire noue-telle avec la société ? Les pratiques scolaires de l'éloquence éclairent-elles ces enjeux épistémologiques et politiques, et réciproquement? Pour tenter de répondre à ces questions, il faut d'abord examiner comment l'éloquence et la rhétorique sont pensées et pratiquées dans l'institution scolaire, et avec quelles finalités. La tâche n'est pas aisée.

2 En premier lieu, parce qu'il est plus difficile qu'aux $\mathrm{XVII}^{\mathrm{e}}$, $\mathrm{XVIII}^{\mathrm{e}}$ et $\mathrm{XIX}^{\mathrm{e}}$ siècles de circonscrire les lieux de l'éloquence comme pratique sociale. Hormis le barreau, les lieux traditionnels de la parole publique migrent de la tribune, de la chaire ou du journal vers internet notamment, où ils se métamorphosent. Une grande enquête comparable à celle que Françoise Douay ${ }^{3}$ mena pour le $\mathrm{xIX}^{\mathrm{e}}$ siècle resterait à entreprendre, pour voir si l'éloquence se renouvelle et se revivifie; et si oui, où et comment. Quoi qu'il en soit, les modèles institutionnels de la parole agissante vacillent. De là, sans doute - et c'est la deuxième difficulté -, l'hétérogénéité des modèles de référence pour son enseignement. En effet, les textes institutionnels (programmes, rapport sur le grand oral de Cyril Delhaye ${ }^{4}$, rapport de l'Inspection générale sur le suivi de l'expérimentation «éloquence » en classe de troisième ${ }^{5}$ ), tout comme les pratiques enseignantes recueillies et observées, révèlent un certain nombre de tensions. Celles-ci se manifestent entre une conception extensive de l'oral qui peine à se définir et une vision restreinte de l'éloquence dont, en outre, la définition est extrêmement labile; 
mais aussi entre divers modèles de parole efficiente, susceptibles eux-mêmes de renvoyer aussi bien à la tradition ancienne des humanités qu'à des références nouvelles. Il y a là un faisceau de questions qui interrogent pour finir la relation de l'enseignement de l'éloquence à celui de l'argumentation et de la littérature et, à ce titre, celle de la place des concours d'éloquence dans cet enseignement. Ce sont ces tensions et ces interrogations que le présent numéro voudrait mettre en lumière ${ }^{6}$.

\section{Le retour de l'éloquence}

3 Avant d'interroger le phénomène lui-même, c'est sur le retour du mot "éloquence " qu'il faut d'abord se pencher, tant il paraît aujourd'hui à la fois imprécis dans ses contours et affaibli dans son contenu, sans doute parce qu'il n'est plus en prise sur la tradition oratoire et littéraire qui lui a donné son sens. On peut d'ailleurs s'étonner de la fortune d'un mot qui pouvait sembler désuet et à peu près sorti de l'usage, pendant toute la deuxième moitié $d u x^{e}$ siècle et encore jusqu'au début des années 2000, pour désigner une pratique et un art de la parole publique. L'étude de Jean Starobinski « La chaire, la tribune, le barreau " qui proposait en 1984 une première synthèse de l'histoire de l'éloquence depuis la Renaissance jusqu'au XIx siècle était d'ailleurs parue dans Les Lieux de mémoire ${ }^{7}$, renvoyant ainsi l'éloquence au passé: elle y évoquait la «figure anachronique » de l'orateur qui, non seulement « n'est plus assigné à son lieu traditionnel » mais « évite assez fréquemment de se montrer en posture d'orateur » et n'est plus l'individu qui, "d'un lieu consacré, rend publics ses convictions, ses arguments, ses sentiments ${ }^{8}$ ». D'autres publications, dans les années 1990, évoquaient également l'éloquence sous le signe de la nostalgie ${ }^{9}$ et le règne de la communication semblait bien avoir renvoyé l'éloquence aux vieilles lunes.

Certes, le mot «éloquence » a un sens courant très large : « Don de la parole, facilité à s'exprimer» ainsi que le définit le Petit Robert avant d'ajouter, cinq lignes après seulement, "Art de toucher et de persuader par le discours ", et le Littré proposait déjà « Facilité à s'exprimer » comme première définition. L'éloquence est aussi couramment définie comme "l'art de bien parler», sans que "bien parler» soit rapporté aux finalités de cette parole ou que le mot "art " renvoie à un ensemble de préceptes précisément circonscrits. Quant aux concours dits d'" éloquence ", auxquels il semble bien qu'on doive le retour en force et en grâce du terme, ils jouent de ce sens à la fois large et imprécis et du prestige de sa grandeur passée. Ainsi, manifestation de ce flou définitoire, le mot renvoie à des formes de parole très éloignées de la tradition du discours oratoire, comme le montre cette présentation des concours Eloquentia - du nom de l'association qui porte également des formations à la prise de parole en public, association popularisée par le film À voix haute ${ }^{10}$ :

Toutes les formes de prise de parole sont admises durant les concours Eloquentia, sans jugement de valeur. Plaidoirie, poésie, rap, stand up ou encore slam, le choix du style de discours se fait à la discrétion des candidats ${ }^{11}$.

Alors que le choix du mot latin semble revendiquer une tradition oratoire remontant à l'Antiquité, les formes de prise de parole énumérées n'ont en commun que le fait de 
relever de la performance orale et gardent peu de rapport avec ce que Cicéron ou Quintilien entendaient par éloquence.

De même, on peut interroger le sens donné au mot au moment où il réapparaît dans le vocabulaire de l'Éducation nationale. Il fait son retour tout d'abord dans l'expérimentation d'un enseignement d'éloquence en classe de troisième, annoncé à la rentrée 2018. Le courrier envoyé aux recteurs en mars 2019 pour en préciser le cadre donne une définition en extension de l'éloquence : «Cet enseignement peut investir tout le champ de l'éloquence : théâtre, lecture en public, arts du spectacle vivant, cinéma, discours, plaidoyer... ». On retrouve sur la page du site Éduscol dédié à l'expérimentation de cet enseignement ${ }^{12}$ une définition tout aussi large qui croise "deux domaines de formation: l'éducation artistique et culturelle, dans ses composantes liées à la parole, et l'apprentissage de l'expression à l'oral ». Il permet ainsi de "travailler l'expression orale continue et l'échange argumenté (débat, plaidoyer...) ainsi que la mise en voix, en geste et en espace de textes littéraires (de la lecture à voix haute à la lecture jouée et au jeu théâtral)». Une acception plus restreinte de l'éloquence semble être proposée puisqu'il est question d'une part du "champ de l'éloquence» et de l'autre des "arts de la parole, qu'elle soit vivante (théâtre, lecture en public, ensemble des arts du spectacle vivant) ou captée (cinémaaudiovisuel). " Mais la partie consacrée aux «enjeux pédagogiques et didactiques » mêle de nouveau toutes les formes de parole en insistant sur leur diversité, parlant tantôt d'" un art de la parole ", tantôt des « arts de la parole » :

La diversité est en effet depuis l'origine une caractéristique de cette éloquence, art commun aux orateurs politiques et aux avocats, aux philosophes, aux acteurs et peut-être même parfois aux poètes. Elle conduit dès lors à cultiver sa parole de façon variée dans des directions fort différentes. Cet enseignement doit ainsi à la fois donner aux élèves quelques repères sur la culture de la parole et sur les arts qui la déploient - jusqu'à cet art sans doute très français de la conversation -, et les mettre en situation d'occuper des positions de parole variées [...].

Et ses vertus sont elles-mêmes diverses puisque la "pratique maitrisée » de la parole doit donner aux élèves « un appui pour mieux s'exprimer, s'adresser aux autres, mais aussi pour progresser dans leur pensée».

7 On semble donc hésiter entre des pratiques artistiques de la parole, et en particulier celle de la parole théatrale, et la parole publique qui vise à agir dans la cité, en y ajoutant également l'art de la conversation, l'ensemble couvrant une part importante du domaine de l'oral tel qu'il figure déjà dans le programme de français. Si toutes ces formes de parole sont évidemment porteuses d'apprentissages importants, elles ne relèvent pourtant pas de la même tradition et n'ont pas la même finalité. D'où l'importance de refaire le point sur ce que recouvre la notion historiquement.

C'est en remontant au latin qu'on pourrait mieux comprendre ce qui s'est joué dans le passage de la tekhnè rhétorikè à l'eloquentia, du rhêtor à l'orator, comme le fait Christian Nicolas à propos de la migration de la terminologie rhétorique du grec au 
latin et au français, montrant comment le système s'enrichit mais aussi se complexifie en conservant en français, dans les termes techniques de la rhétorique, des doublons issus du grec et du latin qu'il s'agit de distinguer et de spécialiser, complexité qui peut faire obstacle lorsqu'on veut étudier la rhétorique et qui invite à retourner aux sources. Mais une telle étude du terme " éloquence » excéderait le cadre de cette introduction. Nous prendrons appui sur les sens du mot dont hérite le $\mathrm{XIX}^{\mathrm{e}}$ siècle pour rappeler qu'alors et jusqu'à la fin du siècle, l'éloquence est à la fois une pratique, une catégorie et un idéal.

9 L'éloquence désigne d'abord en effet la capacité à persuader par le discours. Dans ce sens, tout en lui étant consubstantielle, elle se distingue de la rhétorique dont elle est la pratique, la réalisation dans le discours, ce que résume ainsi l'auteur d'un manuel de rhétorique de la Restauration : «L'Éloquence est l'art de persuader, et la Rhétorique est la théorie de cet $\operatorname{art}^{13}$ ». Autour de ce couple de termes, tourne le débat ancien de la nature et de la technique. L'éloquence est-elle un don, un talent inné ou peut-elle s'enseigner par une technique dont les préceptes sont contenus dans la rhétorique ? La solution consiste en général à dire, depuis Cicéron ${ }^{14}$, que la rhétorique n'a fait que dégager des préceptes en analysant l'éloquence mais que ces préceptes peuvent s'enseigner et permettent d'apprendre plus vite et mieux à bien parler, même s'ils ne peuvent complètement suppléer l'absence de talent naturel ${ }^{15}$. L'éloquence est donc à la fois " un don et un art». Les observations, tirées des ouvrages des maîtres, "sont devenues des règles, sous le nom de rhétorique, et l'art de la parole, né de l'éloquence, a servi ensuite à guider et perfectionner l'éloquence elle-même ${ }^{16} »$. Dans cette acception, l'éloquence est aussi bien celle du discours oral que de l'œuvre écrite.

Comme catégorie littéraire ensuite, l'éloquence se distingue de la poésie, constituant avec elle le "noyau dur» des belles-lettres comme de la littérature (la notion se construit progressivement et prend son sens moderne au xIx siècle) et le fondement des humanités. Elle se subdivise alors en genres, selon la tradition rhétorique : genre délibératif, genre judiciaire et genre démonstratif (ou épidictique), ou, selon une terminologie plus adaptée à la France de l'Ancien Régime : éloquence de la chaifre, éloquence du barreau et, en attendant la renaissance de l'éloquence de la tribune, éloquence académique. Elle englobe parfois, dans les traités, d'autres genres en prose, faute d'une catégorie qui puisse les inclure. Mais dans une délimitation stricte, elle ne désigne que des genres dont la finalité est de persuader.

Enfin, le mot éloquence peut avoir une troisième signification, parfois spécifiée par l'adjectif « véritable » comme dans cette Rhétorique française de Domairon parue en 1804 où, après avoir défini l'éloquence en général comme «le talent de persuader, c'est-àdire de déterminer ceux qui nous écoutent à croire ou à faire quelque chose ", l'auteur en vient à ce qu'il appelle « l'éloquence proprement dite » :

L'éloquence véritable et proprement dite, est comme le pathétique et le vrai sublime; et peut-être ne sont-ils tous les trois qu'une seule et même chose [...]. Le pathétique en effet, est un trait d'une énergie et d'une véhémence extraordinaire qui émeut et agite l'âme; le sublime, un trait d'une noblesse et d'une grandeur 
extraordinaire qui la transporte et l'élève ; l'éloquence, un trait d'une vivacité et d'une rapidité extraordinaire qui la pénètre, la subjugue et la maîtrise ${ }^{17}$. donc s'intéresser à des genres (délibératif, judiciaire, épidictique), à des formes de parole publique et à leur histoire comme à leur devenir dans la période contemporaine. C'est ensuite analyser la mise en œuvre d'un pouvoir de la parole, celui de persuader, en cherchant à retrouver comment les orateurs ont utilisé ou utilisent les préceptes de la rhétorique. Cela suppose de connaître ces préceptes dans toute leur extension, et notamment ceux qui concernent l'invention, et d'apprendre à les mettre en pratique dans des formes de discours où il ne s'agit pas seulement de réaliser une performance orale mais aussi de réfléchir au fond de l'argumentation, à ses moyens et à ses fins. On est donc loin des concours d'éloquence, qui cultivent la virtuosité gratuite du jeu sur les mots, la mise en scène de soi et la punch line en guise de sommet d'éloquence pour obtenir un vote d'adhésion. Quant à l'expérimentation "éloquence» en classe de troisième, on verra dans la contribution d'Alain Brunn que c'est d'abord à une expérience de la parole qu'elle invite et à un parcours dans toutes les possibilités qu'elle offre. Et Nicolas Rouvière, qui a suivi une classe de troisième dans le cadre de cette expérimentation, rend compte d'un projet de mise en voix de textes littéraires qui constitue une première étape dans un parcours qui conduira à inventer et investir sa propre parole.

Plus encore, la réintroduction de ces termes s'effectue à travers un corpus et une terminologie évoquant très fortement le cursus des humanités du XIx ${ }^{e}$ siècle dont, du reste, l'intitulé de l'enseignement de spécialité «Humanités, littérature et philosophie » pourrait être une glose. Rappelons que ce cursus comprenait la classe des humanités (ou seconde), propédeutique à la classe de rhétorique, puis la classe de 
philosophie, qu'il était centré sur l'étude et l'imitation des auteurs grecs et latins, et qu'en classe de rhétorique, les jeunes gens étudiaient " les préceptes de l'art oratoire, lis[ai]ent les orateurs et les historiens et compos[ai]ent force discours en prose [.... ${ }^{19}$ ”. Dans l'enseignement de spécialité, rhétorique et éloquence constituent en elles-mêmes un objet d'étude : sous l'intitulé "l'art de la parole», sont examinés les «pouvoirs", l'«autorité » et les "séductions" de la parole, leur rôle et leurs mises en œuvre à travers un corpus centré sur l'Antiquité et le Moyen Âge qui vise à doter les élèves d'une «culture humaniste ${ }^{20}$ ». La périodisation adoptée manifeste la foi en la transmission, au sens fort du mot, autrement dit en la formation de l'homme dans toute la plénitude du terme, par la fréquentation soutenue de textes portés par une longue tradition.

Quant au corpus proposé, il présente à la fois des aspects conservateurs et novateurs. Le privilège accordé aux auteurs grecs et latins et la part importante des traités de rhétorique ont des allures $\mathrm{XIX}^{\mathrm{e}}$ siècle; cependant, leur approche à travers les trois problématiques des pouvoirs, de l'autorité et des séductions de la parole peut inviter à lire ce corpus comme une reviviscence de la culture des humanités, comme une volonté de retourner aux sources de l'éloquence, dans la tradition humaniste, plutôt que comme un retour en arrière. Il permet notamment de réintroduire dans les programmes scolaires - voire d'introduire, pour la littérature médiévale - des textes qui en avaient été évincés depuis longtemps.

Pour autant, le caractère délibérément très éloigné de ce corpus du point de vue temporel et culturel peut étonner. Certes, le préambule du programme affirme qu'il s'agit de donner aux élèves les moyens de "réfléchir sur les questions contemporaines dans une perspective élargie », et une rubrique " prolongements » suggère la possibilité de mettre le corpus en perspective avec « des exemples d'éloquence parlementaire et politique des époques modernes et contemporaines ${ }^{21} »$. Mais ce lien est présenté comme secondaire. Dès lors, faut-il voir, dans la primauté accordée à la tradition, un manifeste pour une pédagogie du détour? Autrement dit, il s'agirait de demeurer volontairement dans le lointain pour que les élèves puissent, par eux-mêmes, de manière individuelle et apaisée, éclairer des questions contemporaines. Ou alors, ne peut-on pas se demander s'il n'y a pas, là aussi, un effet de neutralisation de l'éloquence?

Dans son article sur les "Pratiques scolaires du discours (1815-1870)22", Corinne Saminadayar-Perrin analyse la «restauration" de l'enseignement de l'éloquence au xix ${ }^{e}$ siècle comme "un dispositif retors pour limiter [s]es possibles dégâts collatéraux ${ }^{23}$ ", à une période (dans les années 1815) où l'on se souvient que les orateurs révolutionnaires ont été formés par les humanités classiques, et où le renouveau du parlementarisme et du barreau d'une part, le sacre romantique de 
l'écrivain d'autre part, replacent la question des pouvoirs de la parole au centre du débat public. Elle montre que dans le milieu scolaire, «il s'agit de garder comme idéal pédagogique, éthique et esthétique la maîtrise du discours, et en même temps de rendre cette pratique de l'éloquence inoffensive hors des murs du collège ${ }^{24}$ ». «Écrire un discours en latin, ajoute-t-elle, c'est en somme poursuivre l'étrange utopie d'une éloquence sans lieu et sans public : se révèlent indirectement (par-là) les inquiétudes attachées à ces pouvoirs de la parole qu'on ne peut exalter qu'en les muselant ${ }^{25}$. »

Dans les programmes du XXI ${ }^{\mathrm{e}}$ siècle, on ne soupçonnera évidemment pas de " dispositif retors ». Néanmoins, la valorisation de l'éloquence semble bien, comme au xix siècle, se doubler d'une crainte à son égard. Cette crainte porterait peut-être tout particulièrement sur « les procédés d'emprise » de la parole - l'expression figure dans les programmes dans la partie concernant « les séductions de la parole »- séductions qui se déclinent d'ailleurs curieusement sous les catégories de l'emprise donc, et de l'amour. Tout se passe comme s'il s'agissait, par le détour des humanités, d'armer les élèves contre diverses formes de stratégies discursives - on voit en filigrane pointer les stratégies de harcèlement ou d'embrigadement idéologique -, sans pour autant les former à l'efficace de la parole, ceci tout en confortant implicitement l'idée que la parole éloquente n'est que le masque d'une parole mensongère, que l'éloquence est nécessairement prise de pouvoir.

On peut alors comprendre le détour par les humanités comme le symptôme de ce qu'Emmanuelle Danblon analyse comme une «crise de l'épidictique " et, en conséquence, de la parole démocratique ${ }^{26}$. Selon elle, l'épidictique est la matrice de tous les genres rhétoriques : reposant sur des valeurs communes, qu'il (ré)actualise dans le même temps, il fonderait la possibilité du désaccord constitutif du délibératif et du judiciaire. Il faut entendre le terme de « désaccord» au sens que lui donne Perelman, c'est-à-dire comme la capacité d'une société, non seulement à accueillir une diversité de points de vue, mais surtout à faire circuler, en tant que telles, ces divergences dans le débat public pour déboucher sur des décisions utiles et éclairées. Le désaccord est donc un signe de santé pour une société et offre une garantie contre la discorde ${ }^{27}$. Or, le détour par les humanités semble destiné à refonder une communauté de valeurs autour d'un corpus actualisant les vertus antiques, les textes des Anciens et les exercices de rhétorique se voyant dotés d'une sorte de performativité morale fantas(ma)tique. En filigrane, l'école, et le programme des humanités en particulier, paraît appelée à restaurer un consensus - et donc, un point de vue unique - autour de certaines valeurs, dans une perspective de paix civile.

21 À côté de ce modèle de parole persuasive s'en dessinent d'autres, purement scolaires. $\mathrm{Au}$ brevet et à l'épreuve anticipée de français au baccalauréat s'ajoute désormais le 
grand oral, qui couronnera le parcours des élèves dans le secondaire et qui se présente explicitement comme un morceau d'éloquence dans le Bulletin officiel:

L'épreuve permet au candidat de montrer sa capacité à prendre la parole en public de façon claire et convaincante. Elle lui permet aussi de mettre les savoirs qu'il a acquis [...] au service d'une argumentation et de montrer comment ces savoirs ont nourri son projet de poursuite d'études $[. .].]^{28}$.

Les indications de cadrage stipulent que le candidat parle debout, et sans notes. Comme le montre Christine Noille, le grand oral signe l'invention d'un nouvel exercice qui, s'il s'appuie sur certaines parties du discours relevant de la tradition rhétorique scolaire (la narratio ou l'expositio), la renouvelle également de manière "déconcertante ». Tout d'abord parce que l'oral y est présenté comme un contrepoint au primat de l'écrit « comme s'il était possible de penser une rhétorique de l'oral coupée de son substrat de réflexion et de préparation qui l'a fondée et motivée à toutes les époques de l'ancienne rhétorique ${ }^{29}$. " L'importance accordée à la parole semble mettre les savoirs au second plan. En second lieu, l'épreuve est complexe. Elle regroupe trois exercices en un seul: une question de spécialité, un "échange avec le candidat» et un échange sur l'orientation du candidat. Or, si le premier et le dernier point peuvent faire l'objet de formalisations techniques autorisant leur apprentissage, le second, en revanche, " est plus ou moins laissé à l'habitus conversationnel du candidat avec des adultes, et [...] à ce titre, il est beaucoup plus élitiste ${ }^{30} »$.

La question de l'enseignement de l'oral se poursuit à l'université, où elle peut bénéficier de programmes spécifiques. C'est le cas par exemple à l'université de Nanterre, où un partenariat a été établi avec l'association Eloquentia dans la perspective du développement de "soft skills» en vue de l'insertion professionnelle. Sans nier l'intérêt de ce type d'apprentissage pour les étudiants, Caroline Facq-Mellet remarque que, fondé sur une parole centrée sur soi et partant de l'écrit, cet oral est très éloigné de celui dont les étudiants ont besoin au cours de leur cursus universitaire. C'est pourquoi elle propose un dispositif alternatif et complémentaire, celui d'une parole argumentative orale fondée sur les interactions en vue de la construction de savoirs scientifiques, qui peut apparaître comme une autre manière de renouveler l'éloquence scolaire.

\section{Éloquence et savoirs}

Le retour de l'éloquence dans l'enseignement pose donc la question des savoirs à mobiliser, et donc potentiellement à enseigner, en particulier dans le cadre du cours de français. Sur quoi prendre appui pour penser une didactique de la parole persuasive? La rhétorique aurait pu constituer ce point d'appui avec ses savoirs théoriques, ses corpus à analyser et ses exercices pratiques. Et Jérôme Lecompte nous montre à quel point le concept rhétorique de l'ethos, qu'on fasse retour vers Aristote ou vers Cicéron et Quintilien pour sa transposition romaine, est à la fois fécond et d'une brûlante actualité pour redonner à l'éloquence une dimension éthique. Mais restée suspecte (et on l'a vu, le programme d'«Humanités, littérature et philosophie " porte encore la trace de cette suspicion), la rhétorique est revenue en partie à travers l'étude de 
l'argumentation, tant du côté de la lecture que de l'écriture ${ }^{31}$. Mais alors que l'argumentation, introduite dans les programmes du collège en 1996, puis du lycée en 2000 comme objet d'étude propre, était encore présente, quoiqu'en recul, dans le programme de français du lycée de 2010, principalement par le prisme des «genres de l'argumentation ${ }^{32}$ ", elle se trouve supplantée, dans le programme de 2019, par l'expression « littérature d'idées » et ne constitue plus un domaine d'étude en tant que tel.

En faisant ce constat, il ne s'agit pas de regretter la place de l'argumentation dans les programmes de 2000 où cette tentative de transposition de diverses approches théoriques relevait d'un bricolage didactique peu satisfaisant et a conduit à une approche essentiellement formelle de l'argumentation ${ }^{33}$. On en veut pour preuve le constat que font dans ce numéro Nathalie Denizot et Laetitia Perret, dans leur étude de la place et du discours scolaire sur Bossuet dans les manuels du XIXe siècle à nos jours : le regain d'intérêt pour l'éloquence suscité par ces programmes n'a pas entraîné de renouvellement de son approche scolaire placée, depuis la fin du xixe siècle, sous le signe du lyrisme et du classicisme, la dimension polémique de son œuvre ayant à peu près disparu. Mais l'absence de l'argumentation telle qu'elle est pensée dans le cadre de la rhétorique, et plus précisément de l'inventio, peut interroger et faire craindre, avec la très grande insistance portée sur l'oral, une prédominance de l'actio aux dépens de la réflexion sur les preuves et sur le raisonnement ${ }^{34}$.

Si le retour de l'éloquence se fait ainsi sans la rhétorique, à laquelle elle était consubstantiellement liée jusqu'au XIX ${ }^{e}$ siècle, un autre modèle de référence est régulièrement convoqué lorsqu'il s'agit d'enseigner l'éloquence. Ainsi, dans le document d'Éduscol consacré à l'expérimentation de l'enseignement «éloquence » en troisième ${ }^{35}$, il est fréquemment fait référence au théâtre décrit comme susceptible d'apporter un modèle, des pratiques, des exercices, à l'articulation entre l'artistique et le culturel, entre l'oral et l'écrit.

Cette référence récurrente se justifie par la place évolutive et grandissante du théâtre dans la sphère de l'éloquence. Au XVII ${ }^{e}$ siècle, comme le rappelle Julia Gros de Gasquet ${ }^{36}$, les trois lieux de l'éloquence, la chaire, le barreau et le théâtre, fonctionnent en écho et mettent en place des outils comparables. La critique s'appuie sur les travaux de Marc Fumaroli qu'elle résume ainsi : "Le théâtre se pose en rival de la chaire et aux côtés du tribunal comme lieu où peuvent raisonner le verbe éloquent et la parole douée d'efficace ${ }^{37}$ ». De fait, le XVII ${ }^{\mathrm{e}}$ siècle place le théâtre sous le double patronage de l'actio et de la rhétorique, comme le montre dans ce numéro, pour cette dernière, l'article de Jérôme Lecompte sur la tragédie classique. Sabine Chaouche, dans L'art du comédien ${ }^{38}$, précise que le mot actio est employé aussi bien pour désigner ce que nous nommons aujourd'hui le " jeu du comédien », que pour qualifier l'animation du discours, par la voix et la gestuelle, des prêtres et des avocats. À partir du xvIII siècle s'opère une disjonction entre les divers arts de la parole qui se traduit par une séparation terminologique. Sabine Chaouche écrit: «Le terme de "déclamation" employé 
spécifiquement pour désigner l'art du comédien, par opposition à l'actio du barreau ou de la chaire, n'apparaîtra qu'au début du XvIII ${ }^{\mathrm{e}}$ siècle lorsque Grimarest dans son Traité du Récitatif ${ }^{39}$, l'emploiera pour distinguer les différents "arts" de la parole (de la simple conversation au chant) et notamment pour mettre en évidence les spécificités propres à chaque "lieu" de la déclamation: discours public du barreau, de la chaire, de l'académie, du théâtre ». À partir du moment où s'élabore un art de l'acteur, la gestuelle et le travail sur la voix seront décrits avec un vocabulaire et des modalités spécifiques - au lieu de l'être à partir de l'orateur. La théâtromanie et la réflexion sur la représentation instaurent au XVIII ${ }^{\mathrm{e}}$ siècle une légitimité du théâtre comme modèle de l'actio, même si évidemment le barreau et la chaire persistent et que la parole politique se développe, prenant un essor notable. Comme l'écrit Laurence Marie dans la quatrième de couverture de son ouvrage Inventer l'acteur : « Avec la fascination exercée par les effets visuels, les comédiens et comédiennes ne sont plus considérés comme de simples déclamateurs, doubles médiocres de l'orateur : ils sont incités à mobiliser tout leur corps pour donner à voir les passions de leur personnage, parfois par-delà les $\operatorname{mots}^{40}$.»

La place du théâtre comme référence dominante se justifie par la constitution progressive d'une identité propre, d'une spécificité pouvant être décrite et transmise. Elle se fonde aussi sur l'effacement d'autres champs d'expression. La sphère politique est désormais convaincue de l'efficacité supposée plus immédiate de la communication; quant à la parole en chaire, si elle existe encore et garde potentiellement sa force, elle reste confinée dans le secret des lieux de culte. Reste, face au théâtre, le barreau qui continue à s'affirmer comme un lieu de référence de l'éloquence, jusque dans les pratiques scolaires ${ }^{41}$. Il est intéressant de noter que s'établit, entre ces deux domaines, une relation de fascination réciproque, qui va de la comparaison entre le prétoire et la scène, au nom d'une spectacularité commune ${ }^{42}$, à la représentation du tribunal sur les plateaux. Mais est-ce une fascination de même nature? On peut faire l'hypothèse que si les avocats empruntent aux acteurs une actio, à savoir une théâtralité qui pourtant existe de moins en moins sur les scènes ${ }^{43}$, le théâtre envie au barreau la parole performative, la force du présent de l'échange ${ }^{44}$, de la dialectique, du débat ${ }^{45}$. Au fond, le théâtre aspirerait à retrouver un espace de débat au moment où la rhétorique s'est perdue dans beaucoup de textes, engloutie dans la disparition progressive de l'action et du personnage. En représentant la parole judiciaire mais aussi les grands marqueurs historiques de la parole politique ${ }^{46}$, le théâtre tenterait de re-présenter une rhétorique à même de lutter contre des phénomènes langagiers de notre époque : la phrase comme unité de pensée, l'opinion comme argumentation. Il établirait un dialogue ${ }^{47}$ fructueux avec l'autre champ dominant de l'éloquence contemporaine, exhibant ainsi les frontières ténues entre la fiction et le réel ${ }^{48}$, autre interrogation fructueuse de la scène d'aujourd'hui.

La référence au théâtre dans la sphère didactique apparaît donc comme légitime au regard de cette évolution. Mais le théâtre est aussi une forme de garant. En effet, étant un art, il évite l'instrumentalisation de l'éloquence à des fins utilitaires, il la protège 
d'une inscription dans la sphère de la communication ou du stand-up. En outre, il est rassurant, car parmi les exemples inscrits par les instructions officielles comme relevant du théâtre, sont décrites des activités déjà pratiquées dans les classes, au risque d'ailleurs que cette compétence intégrative donne lieu à la mise sur le même plan d'exercices dont la nature et les objectifs sont divers. Les pratiques des enseignants ${ }^{49}$, décrites ici par Alain Brunn et par les participants à la table ronde du colloque $^{50}$, montrent de fait que sous le patronage de l'éloquence se regroupent aussi bien la lecture à voix haute de discours que des discussions, des débats, des exposés, des émissions de radio, la participation à des concours d'éloquence, la pratique du slam...

Ces pratiques enseignantes font apparaître des lignes de fracture entre l'oral improvisé et l'oral préparé, entre l'oral et l'écrit oralisé. Elles révèlent aussi une tension entre deux pôles d'idéaux, d'un côté l'acteur, maître de son corps et de sa voix, et l'orateur, pensé comme celui qui parle avec aisance sur un sujet et parvient à convaincre par la force de sa pensée et l'efficacité de sa parole, dont les références, maintes fois convoquées dans les articles de cet ouvrage, seraient les personnages du film À voix haute ou la figure professionnelle de l'avocat du barreau. On peut dégager une autre tension entre deux logiques : d'un côté, l'immersion dans un projet dont on attendrait, par son caractère séduisant et par l'évidence de sa finalité, qu'il fasse surgir des compétences d'éloquence; de l'autre, la progressivité d'une didactique de l'éloquence, souhaitée mais difficile à penser, eu égard au temps imparti à cet enseignement dans le parcours de l'élève, à la lourdeur des groupes et à l'hétérogénéité de leur motivation. Christine Noille, à cet égard, s'étonne que l'on puisse imaginer un enseignement rapide de l'éloquence. S'opposent donc deux représentations : un art de la parole présenté comme un surgissement, lié au contexte théâtral, et un artisanat de la parole, avec des étapes structurées, qui prendrait une valeur artistique au moment de son aboutissement.

Lorsque les enseignants construisent des projets ou participent à ceux que proposent des institutions culturelles, les acteurs sont souvent appelés en renfort, comme dans l'expérience rapportée par Nicolas Rouvière. Or, le risque existe d'attendre de ces partenaires une simple expertise du corps et de la voix alors que les comédiens affirment fréquemment que la technique théâtrale n'est pas déconnectée de la dimension artistique et ne saurait être enseignée en soi et pour soi. Ce que peut apporter le théâtre, dans la didactique de l'éloquence, n'est-ce pas en réalité ce que Pierre Chiron nomme l'érotisme de la parole ${ }^{51}$ ? À partir de cette belle formule se déploie un nouveau champ des possibles qui pourrait déconnecter dans un premier temps l'actio de la rhétorique, pour mieux les réunir ensuite. Il s'agirait, par exemple, de faire expérimenter à l'élève le plaisir physique et sensible de dire des mots. Ainsi, lire ou jouer une liste de noms du théâtre de Valère Novarina amène à la sensation charnelle de trouver sa respiration, de mastiquer des consonnes, de chanter des voyelles. L'érotisme de la parole, c'est aussi incarner une pensée, la faire danser dans son corps. La lecture à voix haute d'un discours réel ou de celui, fictionnel, du personnage de théâtre placé dans une situation où il doit convaincre ou se défendre, 
permet de ressentir physiquement comment on peut se faire traverser par les paroles d'autrui jusqu'à en ressentir une émotion. Reste, pour l'élève, à expérimenter la jubilation née de la production de sa propre pensée. Alors que les pratiques les plus fréquentes disjoignent la recherche d'idées, souvent à l'écrit, et la phase d'oralisation, le théâtre pourrait révéler, en s'appuyant sur les expériences des écrivains de plateau, que de la pensée dite à voix haute peut naître de l'improvisation dirigée, à partir d'une situation bien définie et d'une hypothèse de personnage. Le plus souvent, c'est dans ce présent particulier vécu par l'élève qu'un certain nombre de questions techniques liées au corps et à la voix se posent ou se résolvent naturellement, du fait de la recherche, devenue nécessaire, d'une relation juste avec le public et le partenaire.

Curieusement, c'est peut-être davantage à certaines pratiques d'enseignement de la littérature par l'écriture qu'il revient de cultiver « l'érotisme de la parole ». Dressant un état des lieux des ateliers d'écriture littéraire à l'université, Violaine Houdart-Mérot fait de l'éloquence une clé de l'enseignement-apprentissage de la littérature. Elle montre dans quelle mesure ces expérimentations d'écriture relèvent à la fois de la culture rhétorique (qui consiste à analyser les discours - au sens large - pour mieux en produire) et d'une pratique de l'actio, à travers les lectures qui en sont faites. L'enjeu de ces ateliers est d'entrer dans la littérature par la pratique : écrire pour mieux lire et pour « explorer ce qui se conçoit mal », et inversement.

Dans une optique sensiblement différente, mais qui a toute sa place dans une réflexion sur l'enseignement de l'éloquence et de ses liens avec la littérature, plusieurs contributions montrent que l'éloquence dans la littérature - romanesque - et l'éloquence de la littérature (l'éloquence fonctionnant alors comme outil critique) engagent une réflexion sur le statut et la portée de la parole littéraire. Réfléchie par les textes narratifs, l'éloquence (et non pas uniquement " la rhétorique restreinte ») peut constituer une catégorie d'analyse pertinente, les genres oratoires et la culture rhétorique permettant d'appréhender, dans leur spécificité et dans leur historicité, la dimension esthétique et politique des textes littéraires. Ainsi, après avoir examiné ailleurs comment la littérature se construit comme lieu possible de débat dans le courant $d u \mathrm{XIX}^{\mathrm{e}}$ et au début du $\mathrm{xx}^{\mathrm{e}}$ siècles $^{52}$, Christelle Reggiani analyse ici même la résurgence de l'épidictique sous la forme de l'éloge paradoxal dans la littérature contemporaine - celle du début du xxi siècle - et les enjeux d'une éloquence qui, "[revenant] en farce", se donne à lire comme "humble politique». De son côté, Marta Sukiennicka s'intéresse aux enjeux de la représentation de l'éloquence dans Le Dernier Banquet des Girondins de Nodier, qui caricature l'enseignement qu'il a reçu de la rhétorique "tout en réinventant un nouvel idéal de la parole éloquente », celui d'une parole-action en mode mineur. Enfin, Jawad Tlemsani-Cantin souligne la manière dont Pascal Quignard, faisant revivre l'orateur Albucius et par-là même toute une part oubliée de l'éloquence antique, retrouve dans ses déclamations l'origine même du roman et nous permet de mieux comprendre les voies diverses qu'a pu prendre l'éloquence dans son évolution. 


\section{Renouer avec l'éloquence : pratiques et exercices}

La résurgence de l'éloquence dans les programmes a été précédée par celle des concours d'éloquence : des grandes écoles aux conférences $\mathrm{TEDX}^{53}$ en passant par les établissements scolaires et les associations caritatives, il n'est guère aujourd'hui d'institution qui n'ait le sien. Résurgence là encore, car cette pratique n'est pas neuve. D'emblée, elle entretient des liens ambigus avec l'institution scolaire d'un côté, et la société de l'autre. Ainsi, Stéphane Zékian, qui s'intéresse ici au prix d'éloquence de l'Académie, prestigieux concours qui marqua le XIX siècle, interroge le porte-à-faux de ce prix avec les tendances de l'éloquence qui s'imposent au même moment. Critiqué pour développer une éloquence de commande à date fixe excluant toute inspiration authentique, pour privilégier l'écrit (les candidats envoyaient un texte qu'ils ne disaient pas) et pour tuer l'improvisation, le prix académique est accusé d'être puéril, scolaire, et d'encourager les faiseurs, dans un décalage absolu avec les pratiques et la réflexion contemporaines sur l'éloquence. Au Xxi ${ }^{e}$ siècle, c'est la critique inverse qui prévaut. Il est reproché à l'éloquence valorisée dans les concours d'être, au contraire, trop en phase avec la société : avec certaines formes prisées de communication (le modèle de la battle et de la punch line) et avec des prérogatives d'intégration et de normalisation :

Expression des sans-voix ou maîtrise du verbe dominant, les deux font la paire, avec pour argument clé de la part des organisateurs comme des candidats, les impératifs qu'impose une société de communication: y trouver un travail, et même sa place comme citoyen passerait par la capacité à (se) dire ${ }^{54}$.

C'est l'objet de la critique d'olivier Barbarant ${ }^{55}$, qu'Alain Brunn prolonge avec son analyse du film À voix haute et de la métaphore de la «force de la parole ». En outre, dans les formations à ces concours telles qu'elles sont évoquées dans ce documentaire ou dans le film Le Brio, la rhétorique n'est convoquée que pour quelques catégories et préceptes volontairement simplifiés. Le modèle sous-jacent qui se diffuse est celui des joutes oratoires, des battles, qui appellent indifféremment à plaider le pour et le contre, sur des sujets souvent dérisoires, d'une éloquence sans enjeu véritable, pas plus éthique qu'intellectuel, sinon celui de gagner par tous les moyens. On oublie que l'orateur doit se présenter à son auditoire comme un vir bonus, un homme dont l'autorité tient à sa vertu et à sa rigueur morale, et on ne rappelle pas davantage que les auteurs de traités, depuis Aristote, ont aussi réfléchi sur les fins de la rhétorique, c'est-à-dire sur ce qui oriente le propos de l'orateur: en fonction des genres, ce sera l'utile et l'honnête (ou l'honorable), le juste, ou encore le beau ${ }^{56}$. Ces lieux ou ces valeurs, comme nous pourrions les désigner aujourd'hui, déterminent la ligne argumentative du discours, en affichant les principes invoqués par l'orateur et le parti qu'il défend. Ils sont essentiels tant pour l'analyse des discours (où il s'agit de les identifier) que pour leur élaboration puisqu'ils en déterminent le véritable enjeu.

Pour autant, nombre de contributions de ce dossier insistent sur le besoin d'incarnation du discours et sur la fécondité d'une approche de la rhétorique et de l'éloquence par l'expérience, la notion de rhétorique étant essentielle, le soulignent Emmanuelle Danblon, Lucie Donckier de Donceel, Benoit Sans, Benjamin Sevestre- 
Giraud $^{57}$. En effet, la rhétorique ne saurait se réduire à l'éloquence; son ambition est « bien plus large, puisqu'elle invite [...] à explorer, par la pratique et à travers des outils techniques, le potentiel persuasif du discours et ses modalités logiques, éthiques et pathétiques ».

37 À condition, donc, de penser ensemble éloquence et rhétorique, les concours d'éloquence, comme d'autres exercices oratoires, peuvent développer, chez les élèves et les étudiants, une intelligence pratique rendant sensible le fonctionnement des discours et leurs effets. Guillaume Simiand montre ainsi que le concours d'éloquence, pour peu qu'il soit abordé comme un jeu et pratiqué sur le temps long, permet aux étudiants de droit de s'approprier les éléments d'une culture humaniste éloignée et des techniques disciplinaires $\mathrm{du}$ raisonnement juridique, tout en éprouvant les caractéristiques des trois catégories de preuves du discours. Il insiste sur l'importance de la dimension ludique et sur le processus de décentrement dans la pratique du concours d'éloquence, ainsi que sur la dimension artisanale d'un tel apprentissage. Ces deux aspects sont également soulignés par Anne-Claire f qui analyse les relations entre rhétorique, éloquence et philosophie à travers deux dispositifs didactiques, le colloque et le procès de philosophes. Le caractère ludique de ces deux exercices - nombre d'enseignants interrogés attestent que leurs élèves se « prennent au jeu »- permet aux élèves de mesurer l'impact de savoir-faire discursifs et le plaisir qu'il y a à élaborer une parole agissante en expérimentant « un genre spécifique d'art rhétorique tourné vers la conceptualisation et la problématisation ", le tout en développant des capacités de décentrement. Elle souligne le fait que ce type d'enseignement rhétorique impose une conception élargie de la raison, ouverte à l'intelligence des émotions et à l'intuition. C'est en s'appuyant sur ce postulat, et avec une vocation résolument citoyenne et politique, qu'Emmanuelle Danblon et son équipe du GRAL redonnent vie aux exercices rhétoriques antiques, les progymnasmata. Les chercheurs développent ici un exemple d'actualisation de la suasoire, exercice de déclamation relevant du genre délibératif. Leur pari consiste à partir de la technique rhétorique et d'études de cas, techniques eux aussi (en l'occurrence, médicaux), pour aborder la complexité des processus décisionnels démocratiques. En endossant le rôle du médecin pour construire un discours mettant en jeu les trois preuves (ethos, logos, pathos), les étudiants sont conduits à explorer cette complexité et à éprouver un bon usage de la suspension du jugement, en somme, à développer leur esprit critique.

Si l'on veut penser le retour de l'éloquence dans l'enseignement, il s'agit donc de mieux circonscrire un objet complexe et encore en quête d'un modèle didactique. On pourra alors en cerner les spécificités aussi bien que les enjeux scolaires, sociaux et idéologiques, afin de proposer des orientations pour son enseignement. De l'ensemble varié des contributions qui suivent émergent, du moins l'espère-t-on, quelques lignes de force. Trois nous paraissent particulièrement importantes : la nécessité de penser les voies d'une éthique de la parole ; la remise en cause du modèle judiciaire du tribunal pour enseigner l'éloquence en contexte scolaire, auquel on préférera, avec des visées et des approches différentes selon les auteurs, le modèle délibératif de l'assemblée; la 
voie d'un «érotisme de la parole », qui offre de belles perspectives pour apprendre à penser avec la littérature.

\section{NOTES}

1. «L'Idée d'une réactualisation de la rhétorique est directement liée au rapport qu'une société entretient avec les questions politiques et épistémologiques", entretien avec Emmanuelle Danblon réalisé par Valérie Bonnet, Mots. Les langages du politique, $\mathrm{n}^{0} 125,2021$, p. 111-128.

2. Voir notamment: G. Declercq, "Éloquence et rhétorique au XVII ${ }^{\mathrm{e}}$ siècle en France ", dans M. Prigent, M. Delon, J.-Ch. Darmon (dir.), Histoire de la France littéraire. Clacissismes. XVII ${ }^{2}$ XVIII ${ }^{e}$ siècles, P.U.F., 2006, p. 453-474 ; P. Brasart, "Éloquence et rhétorique au XVIII ${ }^{\mathrm{e}}$ siècle ", ibid., p. 474-491; F. Douay-Soublin, «La Rhétorique en France au XIX ${ }^{\mathrm{e}}$ siècle à travers ses pratiques et ses institutions : restauration, renaissance, remise en cause », dans M. Fumaroli (dir.), Histoire de la rhétorique dans l'Europe moderne (1450-1950), Paris, P.U.F., 1999, p. 1071-1214 ; A. Compagnon, « La réhabilitation de la rhétorique au Xx ${ }^{\mathrm{e}}$ siècle », dans M. Fumaroli (dir.) ouvr. cité, p. 1661-1282.

3. F. Douay-Soublin, art. cité.

4. «Baccalauréat 2021. Faire du grand oral un levier d'égalité des chances. Recommandations pour le grand oral du baccalauréat et l'enseignement de l'oral de la maternelle au lycée », rapport remis à Jean-Michel Blanquer, ministre de l'Éducation nationale et de la jeunesse, le 19 juin 2019.

5. Inspection générale de l'éducation, du sport et de la recherche, Suivi de l'expérimentation d'un enseignement d'éloquence en classe de $3^{e}, \mathrm{n}^{\circ}$ 2021-042, mars 2021.

6. Ce numéro présente les actes du colloque «Penser le retour de l'éloquence dans l'enseignement. Histoire, significations, formes et enjeux » qui s'est tenu à Lyon les 18 et 19 novembre 2020.

7. P. Nora (dir.), Les Lieux de mémoire, t. II, La Nation, vol. 3, Paris, Gallimard, 1984, p. 425-485.

8. Ibid., p. 427.

9. L'Anthologie de l'éloquence française de Jean Calvin à Marguerite Yourcenar de P.Dauzier, P. Lombard (Paris, La Table ronde, 1995) s'ouvrait sur une série d'« images muettes » qui sont tout ce qui reste des «torrents d'éloquence qui se sont déversés sur la France » (p. 9-10); dans Convaincre. Dialogue sur l'éloquence (Paris, éd. Odile Jacob, 1997), J.-D. Bredin et T. Lévy constataient: «L'art oratoire semblait le fondement du métier d'avocat. Il ne l'est plus. [...] Le politique aujourd'hui n'est plus fondé sur l'art oratoire qui est devenu une pratique secondaire. [...] Observons cette étrange évolution : aujourd'hui, où l'accès à l'univers tout entier est possible à travers toutes sortes de médias, la parole ne s'enseigne plus, alors qu'à une époque antique, où l'on restait entre soi, même s'il s'agissait d'exercer les plus hautes fonctions, on ne pouvait pas dissocier la conquête du pouvoir de l'apprentissage de la parole » (p. 34-35).

10. Stéphane De Freitas et Ladj Ly, À voix haute, la force de la parole, 2017.

11. Site du projet Eloquentia : <https://eloquentia.world/les-concours/> [consulté le 19 mai 2021].

12. < https://eduscol.education.fr/630/experimentation-d-un-enseignement-eloquence-en-3e> [consulté le 19 mai 2021].

13. Amar, Cours complet de rhétorique, Paris, Delalain, 1822 ( $3^{\mathrm{e}}$ éd.), p. 143 . Voir aussi la formulation qu'en donne l'article « Rhétorique » de l'Encyclopédie: «La rhétorique est à l'éloquence ce que la théorie est à la pratique, ou comme la poétique est à la poésie. Le rhéteur prescrit des règles 
d'éloquence, l'orateur ou l'homme éloquent fait usage de ces règles pour bien parler; aussi la rhétorique est-elle appelée l'art de parler, et ses règles, règles d'éloquence. » (vol. XIV, p. 250).

14. "Verum ego hanc uim intellego esse in praeceptis omnibus, non ut ea secuti oratores eloquentiae laudem sint adepti, sed, quae sua sponte homines eloquentes facerent. ea quosdam obseruasse atque collegisse; sic esse non eloquentiam ex artificio, sed artificium ex eloquentia natum »: "Toutefois ne nous abusons pas sur la puissance des préceptes : ils n'ont pas formé les grands orateurs; mais on a observé la marche qu'avait suivie le génie guidé par la nature, et on a cherché à suivre ses traces. Ainsi ce n'est pas l'éloquence qui est née de l'art, mais l'art qui est né de l'éloquence » (De Oratore, XXII, 146).

15. Voici cette «solution " telle qu'elle est exposée par Crevier dans sa Rhétorique française de 1767 (Paris, Saillant, Dessaint) : «Tous les Auteurs conviennent que la Rhétorique est née de l'Éloquence. Certains hommes faisaient un meilleur usage que quelques autres du don de la parole: ils traitaient mieux leurs matières: ils se faisaient écouter plus volontiers: ils réussissaient plus sûrement à persuader. Des esprits intelligents et bons observateurs ont remarqué cette différence, \& ils en ont cherché la cause. Ils ont examiné en quoi consistait le mérite supérieur des uns \& le défaut des autres ; ce qui plaisait dans ceux-ci, ce qui rebutait dans ceux-là. La collection de ces observations, comparées avec les principes du raisonnement, \& avec la connaissance du cœur humain, est la Rhétorique. Mais si l'Art est né de l'Éloquence, on voit clairement par sa définition même qu'il sert à perfectionner l'éloquence à son tour. [...] L'Art ne donne point le talent sans doute; mais il l'étend d'une part et le lime de l'autre : il l'avertit de se proportionner à son objet » (Préface).

16. T. Bachelet (dir.), Dictionnaire général des Lettres, des Beaux-Arts et des Sciences morales et politiques, Paris, Delagrave, 1882 ( $1^{\text {re }}$ éd. 1862).

17. Domairon, Rhétorique française, Paris, Deterville, 1804, p. 140. Consultable en ligne sur Gallica : <https://gallica.bnf.fr/ark:/12148/bpt6k9626057g/f11.item.texteImage\#>.

18. « Nul discours ne sera éloquent s'il n'élève l'âme ; [...] On peut donc dire qu'éloquent et sublime sont proprement la même chose », Encyclopédie, article « Élocution » (D’Alembert).

19. F. Douay-Soublin, «Les recueils de discours français pour la classe de rhétorique (XVIII ${ }^{\mathrm{e}}-\mathrm{XIX}{ }^{\mathrm{e}}$ siècles) », Histoire de l'éducation, $\mathrm{n}^{\circ}$ 74, 1997, Les Humanités classiques, p. 152.

20. Bulletin officiel de l'Éducation nationale spécial, $\mathrm{n}^{\circ} 1,1^{\mathrm{er}}$ janvier 2019. Programme d'enseignement de spécialité d'humanités, littérature et philosophie de la classe de première de la voie générale, disponible sur: <https://www.education.gouv.fr/bo/19/Special1/MENE1901578A.htm> [consulté le 12 avril 2020].

21. Les exemples de textes proposés, qui courent d'Érasme à Hugo, n'empêchent pas une ouverture vers cette fameuse « éloquence parlementaire et politique » contemporaine.

22. C. Saminadayar-Perrin, "Pratiques scolaires du discours (1815-1870)», dans H. Millot et C. Saminadayar-Perrin, Spectacles de la parole, Saint-Étienne, Éditions des Cahiers intempestifs, coll. « Lieux littéraires », 2003.

23. Ibid., p. 66.

24. Idem.

25. Ibid., p. 70.

26. E. Danblon, «L'Idée d'une réactualisation de la rhétorique est directement liée au rapport qu'une société entretient avec les questions politiques et épistémologiques », art. cité., p. 119.

27. Entretien avec E. Danblon réalisé par Valérie Bonnet, art. cité, p. 119-120.

28. Bulletin officiel de l'Éducation nationale spécial, $\mathrm{n}^{\circ} 2,13$ février 2020, disponible sur: <https:// www.education.gouv.fr/bo/20/Special2/MENE2002780N.htm> [consulté le 12 avril 2021].

29. Christine Noille, «L'ancienne rhétorique et l'étude des textes », dans ce numéro.

30. Ibid.

31. Pour plus de précisions sur ces relations entre rhétorique et argumentation et leur place dans l'enseignement du français, voir les articles « Argumentation » et « Rhétorique » dans N. Brillant 
Rannou, F. Le Goff, M. - J. Fourtanier, J.-F. Massol, Un Dictionnaire de didactique de la littérature, Paris, Champion, 2020, p. 123-125 et 188-192.

32. En seconde, dans l'objet d'étude "Genres et formes de l'argumentation », on s'intéresse plus particulièrement au développement de l'argumentation, directe ou indirecte; en première, l'objet d'étude s'intitulait « La question de l'Homme dans les genres de l'argumentation du XVI à nos jours ».

33. Voir A. Vibert, «Écriture d'invention et argumentation ", Recherches \& Travaux, $n^{\circ} 73,2008 / 2$, p. 35-87, disponible sur: <https://journals.openedition.org/recherchestravaux/339> [consulté le 5 juin 2021].

34. Fort heureusement, le texte qui définit le grand oral se rapproche davantage d'une tradition qui entend allier un discours fondé sur la raison et une capacité à convaincre : "L'épreuve permet au candidat de montrer sa capacité à prendre la parole en public de façon claire et convaincante. Elle lui permet aussi de mettre les savoirs qu'il a acquis, particulièrement dans ses enseignements de spécialité, au service d'une argumentation, et de montrer comment ces savoirs ont nourri son projet de poursuite d'études, voire son projet professionnel. »

35. Document déjà cité. Voir supra, note 11.

36. J. GROS DE GASQUET, «Rhétorique, théâtralité et corps actorial ", Dix-septième siècle, $\mathrm{n}^{\circ} 236$, 2007/3, p. 501-519. Disponible sur: <https://www.cairn.info/revue-dix-septieme-siecle-2007-3page-501.htm> [consulté le 5 juin 2021].

37. S. Chaouche, L'art du comédien, déclamation et jeu scénique en France à l'âge classique, Paris, Honoré Champion, 2001.

38. Ibid., p. 11.

39. Grimarest commence son traité en donnant une définition du mot « récitatif » qu'il emploie dans son titre : «Le récitatif est l'art de lire, de prononcer ou de chanter un discours, suivant les règles de la prononciation, et de la ponctuation. » J.-L. Le Gallois de Grimarest, Traité du récitatif: dans la lecture, dans l'action publique, dans la déclamation, dans le chant, Paris, J. Le Fèvre et P. Ribou, 1708. Consultable sur Gallica : <https://gallica.bnf.fr/ark:/12148/bpt6k50664w.image>.

40. L. Marie, Inventer l'acteur, émotions et spectacles dans l'Europe des lumières, Paris, Sorbonne Presse Universités, 2019.

41. On peut citer, par exemple, l'exercice du procès du personnage.

42. La comparaison entre la salle du procès et la salle du spectacle est récurrente comme le montre l'ouvrage d'A. Garapon, Bien juger : Essai sur le rituel judiciaire, Paris, Odile Jacob, 2001. Voir aussi G. Soulier : «Le théâtre et le procès », Droit et société, n 17-18, 1991, p. 9-24.

43. L'art de l'acteur de ce début du XxI ${ }^{\mathrm{e}}$ siècle a opéré une mutation vers la parole non projetée. Le non jeu devient une nouvelle norme visant à effacer la distinction entre la parole naturelle et la parole proférée sur les plateaux. Ce phénomène est facilité par l'utilisation des outils technologiques comme le micro qui vient débarrasser la scène de toute artificialité. La voix portée des tribunaux, celle de la parole politique, paraissent contenir une forte théâtralité que revendique moins un théâtre qui susurre à l'oreille des spectateurs.

44. Certains spectacles intègrent des moments de débats avec les spectateurs. On peut citer Un ennemi du peuple d'Ibsen mis en scène par Thomas Ostermeier (2012) ou par Jean-François Sivadier (2019).

45. Cela expliquerait les spectacles récents qui utilisent les procès pour offrir au spectateur un espace de réflexion, de dialectique, comme Mon cœur de Pauline Bureau (2017), ou Olivier Masson doit-il mourir ? écrit par François Hien (2020).

46. Voir la référence aux grands discours $d u$ XVIII $^{\mathrm{e}}$ siècle dans le spectacle Notre terreur de Sylvain Creuzevault (2009).

47. C'est la notion de dialogue qui prévaut si l'on en croit la présentation de l'ouvrage collectif dirigé par Ch. Biet et L. Schifano en 2003, livre qui rassemble des juristes et des universitaires 
spécialistes de théâtre et de cinéma. Voir Représentations du procès : droit, théâtre, littérature, cinéma, Presses universitaires Paris X-Nanterre, coll. « Représentations », 2003.

48. Il est frappant de constater la coexistence temporelle d'un spectacle de Richard Berry Plaidoiries où un acteur interprète les textes de ténors du barreau et À la barre, joué par Éric Dupont-Moretti, devenu depuis garde des sceaux. On peut citer aussi le spectacle Please, continue (Hamlet), de Roger Bernat et Yan Duyvendak, qui invitait tous les soirs des professionnels du barreau dans un procès fictif du personnage de Shakespeare (TNP, 2014).

49. Comme en témoigne le questionnaire rempli par les participants lors d'un stage organisé par la DAAC de l'académie de Lyon et animé par Anne Fournier et David Rignault.

50. Cette table ronde a réuni des personnalités lyonnaises : Valérie Perrin, déléguée académique aux Arts et la Culture, Vincent Tuleu, IA-IPR de Lettres, Didier Richard, responsable des publics du Théâtre des Célestins.

51. Dans "Être et avoir", sur France Culture, émission de Louise Touret du 20/05/2020, «Enseigner l'éloquence pour redonner la parole»: <https://www.franceculture.fr/emissions/ etre-et-savoir/enseigner-leloquence-pour-redonner-la-parole> [consulté le 5 juin 2021].

52. C. Reggiani, Éloquence du roman. Rhétorique, littérature et politique aux XIX $X^{e}$ et $X X^{e}$ siècles, Genève, Droz, 2008.

53. Les conférences TED (Technology, Entertainment and Design), organisées par l'association à but non lucratif The Sapling Foundation, se donnent pour but, selon leur slogan, de «diffuser les idées qui en valent la peine ». Voir: <https://www.ted.com/talks?language=fr> [consulté le 12 avril 2020].

54. O. Barbarant, « L'éloquence ou le bagout? », Le Monde diplomatique, novembre 2018.

55. Ibid.

56. Encore appelés lieux «du souverain bien », ils donnent une finalité à l'argumentation. Ils figurent dans la liste des sept lieux que donne Aphtonius pour l'exercice du lieu commun : «Les derniers points de cet exercice sont les arguments finaux (telika kephalaia; en lat. capita finalia) : le légal, le juste, l'utile, le possible, l'honorable, les conséquences futures ». Cité par F. Goyet, Le sublime du " lieu commun ». L'invention rhétorique dans l'Antiquité et à la Renaissance, Paris, Champion, 1996, p. 91.

57. Emmanuelle Danblon, Lucie Donckier de Donceel, Benoît Sans et Benjamin Sevestre-Giraud, « Enseigner la rhétorique à l'École de Bruxelles », dans ce numéro.

\section{RÉSUMÉS}

Si on assiste à une revalorisation de l'éloquence, dont témoignent la vogue des concours d'éloquence, le succès d'un film comme À voix haute et la réévaluation de l'oral et des arts de la parole dans le parcours des élèves du collège au lycée (expérimentation d'un enseignement d'«éloquence » en troisième, "grand oral» au baccalauréat), les objectifs assignés à son enseignement, à l'école et à l'université, apparaissent encore très hétérogènes. Considérant l'éloquence comme un enjeu éducatif et politique fondamental, en relation étroite avec des pratiques sociales en mutation, les textes réunis ici interrogent les cadres théoriques qui doivent permettre de fonder une didactique de la parole orale, ainsi que des expériences d'enseignement de l'éloquence et leurs enjeux. 
Considering a revaluation of eloquence, as witnessed by the vogue for eloquence contests, the success of a film such as À voix haute and the revaluation of oral and speech arts in the course of students from junior high school to high school (eloquence experimentation in $9^{\text {th }}$ grade, "grand oral" in the A-levels), the objectives assigned to its teaching, at school and university, still appear to be very heterogeneous. Considering eloquence as a fundamental educational and political issue, in close relation with changing social practices, the texts gathered here question the theoretical frameworks that should make it possible to found a didactic of oral speech, as well as experiences of teaching eloquence and their stakes.

\section{INDEX}

Keywords : eloquence, oral, patterns, practices, teaching

Mots-clés : éloquence, oral, modèles, pratiques, enseignement

\section{AUTEURS}

\section{MARION MAS}

Université Lyon 1 (INSPÉ) - IHRIM UMR 5317

Marion Mas est maîtresse de conférences en littérature française à l'INSPÉ de Lyon et membre de l'IHRIM (UMR 5317). Spécialiste de Balzac, elle a consacré plusieurs articles à cet auteur, ainsi qu'un livre issu de sa thèse : Le Père Balzac. Représentations de la paternité dans La Comédie humaine (Classiques-Garnier, 2015). Actuellement, ses travaux portent d'une part sur les relations entre droit et littérature et d'autre part sur l'enseignement de la littérature. Elle a récemment publié : Le Code en toute lettres : écritures et réécritures du Code civil au XIX ${ }^{e}$ siècle (en co-direction avec François Kerlouégan ; Classiques-Garnier, 2020) ; « La littérature au corps : expérience sensible du littéraire et engagement éthique dans les concours d'éloquence », dans N. Brillant Rannou, F. Le Goff \& M. Sauvaire (dir.), Expérience et partage du sensible dans l'enseignement de la littérature. Actes des $20^{e}$ Rencontres des chercheurs en didactique de la littérature de Rennes, à paraître en ligne sur le site de Sciences Conf, CCSD cnrs.fr. ; « Une littérature féminine? analyse du discours sur la lecture de littérature à travers un corpus d'anthologies pour jeunes filles, de la monarchie de Juillet à la Troisième République » (Les Cahiers FabLiJeS, $n^{\circ}$ 1, à paraître).

\section{CATHERINE NICOLAS}

Université Lyon 1 (INSPÉ) - IHRIM UMR 5317

Catherine Ailloud-Nicolas est maîtresse de conférences à l'INSPÉ de Lyon (université Claude Bernard-Lyon 1) et membre de l'IHRIM. Spécialiste de Marivaux, elle a édité Le Legs et a écrit avec Christelle Bahier-Porte une étude intitulée Dramaturgies de l'inconstance. Elle prépare actuellement une dizaine d'articles pour le Dictionnaire de Marivaux, en cours d'élaboration. Par ailleurs, elle travaille en tant que dramaturge dans de nombreux spectacles de théâtre et d'opéras, en particulier pour Richard Brunel, directeur de l'Opéra de Lyon. Elle enseigne la dramaturgie auprès des comédiens du Conservatoire de Lyon et auprès des créateurs de l'ENSATT, et en propose une transposition didactique pour les enseignants, dans des articles parus ou à paraître.

\section{ANNE VIBERT}

IGESR, Litt\&arts - UMR 5316

Anne Vibert est inspectrice générale de l'Éducation du sport et de la recherche et membre 
associée de Litt\&arts - UMR 5316, Université Grenoble-Alpes. Auteure d'une thèse intitulée L'éloquence parlementaire sous la Monarchie de Juillet. Guizot, Thiers, Tocqueville (2000), elle était antérieurement maitresse de conférences en littérature du XIX ${ }^{\mathrm{e}}$ siècle. Elle a dirigé un ouvrage sur l'éloquence judiciaire (L'Éloquence judiciaire. Préceptes et pratiques. Grandes plaidoiries passées et contemporaines, Paris, Litec/Éditions du Juris-Classeur, 2003) et publié plusieurs articles sur l'éloquence et la rhétorique, parmi lesquels « L'éloquence révolutionnaire : modèle ou contremodèle pour l'éloquence politique du $\mathrm{XIX}^{\mathrm{e}}$ siècle ?» (dans Une expérience rhétorique. L'éloquence de la Révolution, É. Négrel et J.-P. Sermain éd., Oxford, Voltaire Foundation, 2002) ; « Fontanier : autour et au-delà. La rhétorique dans le premier tiers du XIX ${ }^{\mathrm{e}}$ siècle » $\left(R H L F, \mathrm{n}^{\circ} 2,2005\right)$;

«L'impression tue l'éloquence » (Lieux littéraires/La Revue, $\mathrm{n}^{\circ}$ 11-12, 2010), ainsi que des articles portant sur leur didactique et celle de la littérature d'idées (« Actualité de la rhétorique : pour une propédeutique de l'oral ", dans Écoute mon papyrus. Littérature, oral et oralité, Ph. Clermont et A. Schneider (dir.), Strasbourg, Scéren-CRDP d'Alsace, 2006 ; « Écriture d'invention et argumentation ", Recherches \& Travaux, $\mathrm{n}^{\circ} 73,2008 / 2$, « La littérature d'idées appartient-elle à la culture commune? », avec G. Plissonneau, dans I. de Peretti et B. Ferrier (dir.), Enseigner les " classiques » aujourd'hui. Approches critiques et didactiques, Bruxelles, Peter Lang, 2012). Elle vient de faire paraitre en collaboration avec Françoise Mélonio le tome XVII des CEuvres complètes de Tocqueville, Correspondance et écrits divers, aux éditions Gallimard (3 volumes, juin 2021). 